\title{
Ingo Winkler
}

\section{Intentionen und Problemlagen der Nutzung atypischer Beschäftigung im ostdeutschen Mittelstand}

\begin{abstract}
Der vorliegende Beitrag beschäftigt sich mit der Nutzung atypischer Beschäftigung im ostdeutschen Mittelstand. Basierend auf den Ergebnissen einer explorativen Fragebogenuntersuchung werden zunächst die Nutzungsformen und die Nutzungsintensität von Leiharbeit, geringfügiger Beschäftigung, Teilzeitarbeit sowie Telearbeit untersucht. Hierbei lassen die Ergebnisse den Rückschluss zu, dass sich atypische Beschäftigungsformen in ostdeutschen KMU etabliert haben. Daran anschließend werden die Zielvorstellungen der Geschäftsleitungen dargestellt, die die Nutzung atypischer Beschäftigung leiten. Diese sind vorwiegend ökonomischer Natur. Hierbei wird auch die Wahrnehmung der Zielerreichung analysiert. Weiterhin wird auf Probleme fokussiert, die mit der Nutzung atypischer Beschäftigung in den untersuchten KMU auftreten. Dabei werden Antworten von Geschäftsleitungen und Arbeitnehmervertretern verglichen und Unterschiede herausgestellt. Es zeigt sich, dass Arbeitnehmervertreter mehr und zum Teil auch andere Probleme hervorheben als die Geschäftsleitungen.
\end{abstract}

\section{$1 \quad$ Einleitung}

Nachdem atypische Beschäftigung in großen Unternehmen schon seit geraumer Zeit nichts Neues ist, nutzen auch kleine und mittelständische Unternehmen (KMU) diese Art der Beschäftigung. Da KMU sich häufig in einer erschwerten Wettbewerbssituation befinden und der Kosten- und Flexibilitätsdruck weiter steigt, erscheinen die verschiedenen Formen atypischer Beschäftigung, wie z.B. Leiharbeit, Minijobs oder Teilzeitbeschäftigung, ein probates Mittel, diesen Problemen zu begegnen. Dabei weisen ostdeutsche KMU häufig eine höhere Nutzungsintensität auf (Schäfer 2000, 46; Düll/Ellguth 1999, 279), was zum Teil mit ihrer prekären wirtschaftlichen Situation begründet wird.

Der vorliegende Beitrag stellt die Ergebnisse einer explorativen empirischen Erhebung zu atypischer Beschäftigung in KMU in Sachsen sowie den benachbarten Bundesländern vor. Hierbei wird, über die bisherige Forschung hinaus, auf Ziele sowie Probleme der Firmen bei der Nutzung verschiedener Formen atypischer Beschäftigung fokussiert. Zudem wird für die Ergebnisse der Fragebogenaktion eine Reihe von Interpretationsmöglichkeiten dargestellt.

Im folgenden Abschnitt werden zunächst die Ergebnisse der bisherigen Forschung zu atypischer Beschäftigung in KMU sowie speziell in ostdeutschen KMU reflektiert. Daran anschließend erfolgt die Darstellung der beiden forschungsleitenden Fragestellungen. Nach der Beschreibung der Stichprobe sowie der Untersuchungsmethode werden die Ergebnisse präsentiert. Diese werden hinsichtlich möglicher Ursachen oder Konsequenzen interpretiert. Der Beitrag schließt mit einer Zusammenfassung der wichtigsten Ergebnisse sowie einem kurzen Ausblick zu sich ergebenden weiteren Forschungsbedarfen.

Arbeit, Heft 3, Jg 14 (2005), S. 207-220 


\section{Atypische Beschäftigung in kleinen und mittleren Unternehmen}

In der BRD ist bei KMU eine steigende Nutzungshäufigkeit atypischer Beschäftigung festzustellen, wogegen bei großen Unternehmen eher die gegenläufige Tendenz zu verzeichnen ist (Rudolph/Schröder 1997, 111; Düll/Ellguth 1999, 279; Krömmelbein 2000, 140). In diesem Zusammenhang ist auch in der mittelständischen Wirtschaft eine tendenzielle Abnahme von Normalarbeitsverhältnissen zu beobachten (Bellmann/Dahms/Wahse 2004, 40).

Betrachtet man die Gründe für das vermehrte Auftreten atypischer Beschäftigung in KMU, so werden diese einerseits darin gesehen, dass hier, ähnlich wie in großen Unternehmen, positive betriebswirtschaftliche Wirkungen zu erwarten sind, insbesondere erhöhte numerische Flexibilität sowie Kostenvorteile (Brewster/Hegewisch/Mayne 1994, 170; Nienhüser/ Baumhus 2002, 75; Corral/Isusi 2003, 2). ${ }^{1}$

Andererseits wird argumentiert, dass diese Beschäftigungsformen insbesondere bei neu gegründeten oder jungen Unternehmen zu finden sind und diese sind typischerweise klein oder mittelständisch (Hoffmann/Walwei 1998, 419; Schäfer 2000, 46). Cardon (2003) versteht in diesem Zusammenhang atypische Beschäftigung als Möglichkeit, das Wachstum neu gegründeter KMU zu unterstützen. In den einzelnen Entwicklungsphasen der Unternehmen (Startup, Expansion, Reife, Diversifikation) sind dabei unterschiedliche Formen atypischer Beschäftigung sinnvoll (Cardon 2003, 363).

Darüber hinaus kann man in Anlehnung an Mayne u.a. $(2000,72)$ eine Art institutionellen Isomorphismus vermuten. KMU imitieren, auch wegen der legitimierenden Flankierung durch die wissenschaftliche und journalistische Thematisierung (Martin/Nienhüser 2002, 1) und der gesellschaftlichen Akzeptanz atypischer Beschäftigung (Grimm 2004), die anscheinend erfolgreichen Strategien großer Unternehmen beim Umgang mit verschärftem Wettbewerb.

Betrachtet man Ost-West-Unterschiede, so nutzen, insgesamt betrachtet, ostdeutsche Betriebe atypische Formen der Beschäftigung in stärkerem Umfang (ohne Autor 2000, 4; Schäfer 2000, 46; Düll/Ellguth 1999, 279), wobei je nach Beschäftigungsform auch differenzierte Entwicklungen zu beobachten sind (Bellmann/Dahms/Wahse 2004, 40; Hoffmann/ Walwei 1998, 418; IAB-Betriebspanel Ost 2003, 28ff.; Voss-Dahm 2004, 359). Die stärkere Nutzung in Ostdeutschland wird unter anderem mit der größeren wirtschaftlichen Unsicherheit bei den Betrieben in der Umbruchsituation sowie weit verbreiteten ABM-Maßnahmen begründet (ohne Autor 2000, 5; Schäfer 2000, 46; Vogel 1999, 16). Zudem wird teilweise argumentiert, dass in ostdeutschen Betrieben, aufgrund der mit der Wende verbundenen tief greifenden Transformationsprozesse, zukünftige Produktionsmodelle quasi vorweggenommen werden (Brinkmann 1996; Brinkmann 2003).

Die bisherige Forschung zu atypischer Beschäftigung in KMU beschränkt sich jedoch weitgehend auf statistische Erhebungen zur Nutzungshäufigkeit und -intensität.

Potentielle Vorteile der Nutzung für die Unternehmen werden nur selten postuliert. Implizit werden Kosten- und Flexibilitätsvorteilen angenommen, spezifische Betrachtungen

1 Insgesamt zeigt die deutschsprachige Diskussion zu atypischer Beschäftigung eine deutliche Betonung von Kosten- und Flexibilitätskriterien. Andere Ziele, Formen atypische Beschäftigung zu nutzen, wie z.B. das Halten ausgezeichneter Mitarbeiter, indem ihnen Flexibilisierungsmöglichkeiten eingeräumt werden (Tilly 1992), oder atypisch Beschäftigte als Wissensressource (Matusik/Hill 1998; Foote/Folta 2002), finden sich fast ausschließlich in der internationalen Diskussion. 
für KMU, die eventuell über betriebswirtschaftliche Effizienzkriterien hinausgehen, finden bisher nicht statt.

Weiterhin sind mögliche spezifische betriebliche Probleme der Nutzung atypischer Beschäftigung in KMU bisher nicht Gegenstand der Forschung. Vielmehr werden, ebenfalls häufig implizit, allgemeine Probleme für Unternehmen und Beschäftigte auch auf diesen Sektor übertragen. Insbesondere wird angenommen, dass Anpassungs- und Lernprobleme (Martin 2002, 505), Integrationsprobleme (Becker 1998, 106), Qualitätsprobleme (Nienhüser/Baumhus 2002, 99) oder Vertrauensverluste (Pearce 1993, 1090; Seifert/Pawlowsky 1998, 604) sich auch bei atypischer Beschäftigung im KMU finden lassen. Spezifische empirische Untersuchungen, die diese Vermutung überprüfen oder differenzierte Aspekte liefern, fehlen jedoch.

Insgesamt besteht hier erheblicher Forschungsbedarf, wobei sich u.a. folgende Fragen stellen lassen: Warum nutzen KMU atypische Beschäftigungsformen? Werden bestimmte Formen bevorzugt und weshalb? Welche konkreten Probleme ergeben sich für das Management und die Mitarbeiter dieser Unternehmen? So ist z.B. anzunehmen, dass KMU häufiger Probleme mit Zeitarbeitsfirmen haben, da sie, wegen der oft geringen Entleihmenge, keine ausgeprägte Machtposition gegenüber Verleihern besitzen. Weiterhin kann erwartet werden, dass der i.d.R. höhere Bedarf an motivierten Mitarbeitern in Generalistenfunktion verstärkt Probleme bei der Nutzung atypischer Beschäftigung hervorruft. Darüber hinaus können die häufig ausgeprägten sozialen Beziehungen zwischen den Mitarbeitern in KMU einerseits dazu führen, dass atypisch Beschäftigte zu unterdrückten Außenseitern werden, und andererseits kann der Einsatz von Fremdarbeitskräften diese Beziehungen selbst empfindlich stören.

\section{Forschungsfragen}

Der vorliegende Beitrag beschäftigt sich mit einem Teil der genannten Problemfelder. Auf der Basis einer empirischen Untersuchung in ostdeutschen $\mathrm{KMU}^{2}$ wird insbesondere auf Nutzungsformen und -häufigkeiten sowie Ziele und Problemlagen fokussiert. Die Gründe für die Konzentration auf ostdeutsche Unternehmen, liegen einerseits in der regionalen Lage der Universität, an der die Untersuchung durchgeführt wurde. Andererseits sind in Ostdeutschland überwiegend kleine und mittelgroße Betriebe zu finden, deren Situation allgemein als prekär eingeschätzt wird ${ }^{3}$, was eine verstärkte Nutzung atypischer Formen der Beschäftigung zur Bewältigung der schwierigen Unternehmenssituation vermuten lässt. Hinzu kommt, dass in den neuen Bundesländern mit der Zunahme atypischer Beschäftigung gleichzeitig ein Rückgang klassischer Arbeitsverhältnisse verbunden ist (Bertelsmann Stiftung, Hans-Böckler-Stiftung (Hg.) 1998, 4; Schäfer 2000, 46).

Basierend auf den Ergebnissen der Untersuchung soll ein erstes Bild zu atypischer Beschäftigung im ostdeutschen Mittelstand entworfen werden, wobei zwei Themenschwerpunkte gesetzt werden:

- Erstens werden die Ziele untersucht, welche die Geschäftsleitungen mit der Einführung/ Nutzung verbinden. Angenommen wurde hierbei, dass diese auch bei den Geschäfts-

2 In der Untersuchung werden KMU quantitativ, anhand der Mitarbeiterzahl von anderen Unternehmen abgegrenzt. Als klein und mittelständisch werden Unternehmen mit 20 bis 1000 Mitarbeitern definiert.

3 So gab im IAB-Betriebspanel Ost 2004 jedes dritte ostdeutsche Unternehmen an, im Jahre 2002 von stärkeren Schwankungen in der Produktions- und Geschäftstätigkeit betroffen gewesen zu sein (Westdeutschland 27\%) (Bellmann/Dahms/Wahse 2004, 32). 
leitungen von KMU vorwiegend ökonomischer Natur sind. Diese Vermutung basiert zum einen auf der allgemeinen Argumentation in der Literatur, zum anderen ist vom Management eine solche rationale Begründung zu erwarten. Gleichwohl wurden in die Antwortvorgaben auch Ziele aufgenommen, die nicht primär als ökonomisch zu bezeichnen sind.

- Zweitens werden Probleme adressiert, die mit der Nutzung atypischer Formen der Beschäftigung in den befragten Unternehmen auftreten. Hier wurde den Befragten nahezu der gesamte, aus der Literatur bekannte Kanon an möglichen Problemen präsentiert. Innerhalb dieses Schwerpunktes werden die Antworten aus den Managementfragebögen mit Antworten aus spezifischen Fragebögen für Arbeitnehmervertreter verglichen. Angenommen wurde hierbei, dass die Arbeitnehmervertreter eine kritischere Einschätzung vornehmen und u.U. andere Probleme betonen, als die Geschäftsleitungen der Betriebe. Neben den definierten Antwortvorgaben wurde auch Raum für offene Antwortmöglichkeiten gelassen. Somit hatten die Befragten prinzipiell die Möglichkeit, eigene, u.U. KMU-spezifische Angaben zu machen.

Obwohl die Untersuchung in ostdeutschen KMU durchgeführt wurde, kann angenommen werden, dass die Ergebnisse auch Erklärungsansätze für den gesamten KMU-Sektor liefern. So ist zu vermuten, dass die Zielvorstellungen, unter denen atypische Formen der Beschäftigung genutzt werden, sowie die auftretenden Probleme, so oder ähnlich in vielen deutschen KMU zu finden sind.

\section{$4 \quad$ Stichprobe und Methode}

Die vorliegende explorative Querschnittsuntersuchung basiert auf einem zweiseitigen Fragebogen, der an die Geschäftsleitungen von 736 kleinen und mittelständischen Firmen (zwischen 20 - 1000 Mitarbeiter) in Sachsen sowie den angrenzenden Bundesländern gesendet wurde. Die Adressen der Erhebung wurden aus der Datenbank „ABC der deutschen Wirtschaft“ generiert. Von den 288 zurück erhaltenen Fragebögen (Rücklaufquote 39,1\%) wurden alle Fragebögen in die Auswertung einbezogen.

Der Großteil dieser Fragebögen kam aus Sachsen (168). Der Rest verteilte sich auf Sachsen-Anhalt (34), Thüringen (41) sowie Brandenburg (8). Somit weisen die in die Auswertung einbezogenen Fragebögen einen Schwerpunkt bezüglich sächsischer KMU auf.

Darüber hinaus wurden in die Auswertung 40 Fragebögen einbezogen, die von Arbeitnehmervertretern in sächsischen Unternehmen ausgefüllt wurden. Diese werden insbesondere bei der Auswertung möglicher Probleme mit atypischer Beschäftigung herangezogen und den Antworten der Geschäftsleitungen gegenübergestellt.

Die Erhebung fokussierte auf vier Formen atypischer Beschäftigung:

- Leiharbeit (Arbeitskräfte einer Leiharbeit- oder Zeitarbeitsfirma)

- abhängige Teilzeitbeschäftigung (Mitarbeiter, die zeitweise oder dauerhaft verkürzt arbeiten)

- geringfügige Beschäftigung (so genannte Mini-Jobs mit bis zu 400 Euro Verdienst)

- Telearbeit (Arbeitskräfte, die vorwiegend zu Hause unter Nutzung von Computern arbeiten und mit dem Unternehmen i.d.R. über Telefon, Fax oder per Internet kommunizieren)

Diese Beschränkung erfolgte insbesondere unter der Annahme, dass diese Formen in den Betrieben am häufigsten genutzt werden und ihnen somit bekannt sind. Zusätzlich hatten die 
Betriebe jedoch auch die Möglichkeit, andere Formen atypischer Beschäftigung, z.B. so genannte Midi-Jobs, zu nennen.

\section{$5 \quad$ Ergebnisse und Diskussion}

\subsection{Nutzungsformen und Nutzungsintensität}

Betrachtet man die Nutzung atypischer Beschäftigung im ostdeutschen Mittelstand ${ }^{4}$ so zeigt sich, dass der überwiegende Teil der befragten Unternehmen (87,5\%) atypische Beschäftigungsformen nutzen. Diejenigen Unternehmen, die dies nicht tun (12,3\%), gaben als Gründe an, dass sie spezifische Qualifikationen bei ihren Mitarbeitern benötigen. Über diese verfügen nach Meinung dieser Geschäftsleitungen atypisch Beschäftigte nicht bzw. scheint es schwer bis unmöglich, diesen Beschäftigten solche Qualifikationen zu vermitteln. Zudem wurde angegeben, dass bei atypischer Beschäftigung die Nachteile für die Unternehmen überwiegen, was vermuten lässt, dass die häufig genannten Vorteile für die Nutzung dieser Beschäftigungsformen, wie numerische Flexibilität und Kosteneinsparungen, aus Sicht dieser Unternehmen nicht realisiert werden.

Die Auswertung der Frage nach den Formen atypischer Beschäftigung, die von den befragten Firmen genutzt werden, ergab, dass Leih- oder Zeitarbeit mit 37,9\% die dominante Form atypischer Beschäftigung im ostdeutschen Mittelstand ist. Geringfügige Beschäftigung (31,9\%) und abhängige Teilzeitbeschäftigung (24,3\%) bilden das Mittelfeld der Aussagen. Die geringere Nutzung dieser Formen kann auf die fehlende Quasi-Garantie der fachlichen Eignung der Beschäftigten (Nienhüser/Baumhus 2002, 84) zurückgeführt werden, die bei Zeitarbeit i.d.R. unterstellt wird. Somit müssen Teilzeit- und geringfügig Beschäftigte erst angelernt bzw. eingearbeitet werden. Zudem ist hier der Such- und Auswahlprozess für die Firmen aufwändiger.

Telearbeit wird in den befragten KMU kaum genutzt (2,7\%). Dieses Resultat zeigt einen deutlichen Unterschied zu Krömmelbein (2002, 140), die in ihrer Studie westdeutscher Unternehmen in der Region Rhein-Main die höchste Nutzung von Telearbeit bei der Betriebsgrößenklasse 50-499 Mitarbeiter festgestellt hat. Die in der vorliegenden Untersuchung festgestellte geringe Nutzung in ostdeutschen Betrieben hängt einerseits damit zusammen, dass die mit Telearbeit verbundene notwendig gute Ausstattung der Unternehmen und der Telearbeiter mit IuK-Technologien für ostdeutsche KMU eine hohe finanzielle Hürde darstellt. Andererseits bedeutet insbesondere Vollzeit-Telearbeit, dass die Beschäftigten nur selten im Unternehmen präsent sind. Dies ist jedoch nicht passfähig zu dem häufig ausgeprägten Zentralisierungs- und Kontrollverhalten der Inhaber kleiner und mittlerer Unternehmen in Ostdeutschland (Meinerz 1996; Alt/Lang 1997; 1998). So erfordert Telearbeit zum Teil ein anderes Verhalten von Vorgesetzten, da direkte Beobachtung und Kontrolle nicht möglich sind (Dimitrova 2003, 191; Topi 2003, 81).

Als andere Formen atypischer Beschäftigung (3,1\%) wurden u.a. kurzfristige Tätigkeiten von Schülern und Studenten, Werksstudenten sowie Heimarbeiter genannt.

Betrachtet man den Anteil, den die einzelnen Formen atypischer Beschäftigung an der Gesamtzahl der Mitarbeiter der Unternehmen haben, so zeigt sich deutlich, dass in den untersuchten Betrieben so genannte Normalarbeitsverhältnisse nicht durch atypische Beschäftigung ersetzt werden. So werden in den meisten Unternehmen weniger als $10 \%$ der 4 Die von den Firmen angegebenen Mitarbeiterzahlen zeigen mit 5 - 900 Beschäftigten ein breites Spektrum. 
gesamten Mitarbeiterzahl in atypischer Beschäftigung eingesetzt. Der geringe Prozentsatz der Nutzung deutet darauf hin, dass diese Beschäftigungsformen im ostdeutschen Mittelstand als Ergänzung genutzt werden. Die befragten Unternehmen erhalten sich eine Kernbelegschaft, um die eine Peripherie aus flexiblen Beschäftigungsformen aufgebaut wird. ${ }^{5}$

Atypische Beschäftigung wird von den befragten Unternehmen vorwiegend im Produktionsbereich genutzt $(67,4 \%)$, wobei es sich hier i.d.R. um Leiharbeiter handelt. Nur ein geringer Prozentsatz der Betriebe nutzt diese Beschäftigungsformen in der Verwaltung (4,2\%), dabei häufig geringfügig Beschäftigte. Zum Teil werden atypische Beschäftigte auch in beiden Bereichen eingesetzt (15,6\%). ${ }^{6}$ Aus dieser Verteilung lässt sich einerseits ableiten, dass sich der Produktionsbereich offensichtlich am einfachsten in Teilprozesse und -aufgaben zerlegen lässt, die dann durch flexible Beschäftigungsformen realisiert werden. Andererseits wird dieser Bereich vom Management quasi als Manövriermasse betrachtet, um Auftragsschwankungen aufzufangen und Kostenvorteile zu erzielen. Jedoch wird dies auch der Kernbelegschaft in diesem Bereich klar, insbesondere die mehr oder weniger permanente Bedrohung ihrer Arbeitsplätze, wenn mehr Flexibilität erforderlich wird. Gerade im Produktionsbereich können demnach die meisten Probleme hinsichtlich des in der Literatur angeführten Vertrauensverlustes der Stammbelegschaft gegenüber der Geschäftsleitung erwartet werden.

\subsection{Nutzung in Abhängigkeit von der Mitarbeiterzahl}

\section{Abb. 1: Mitarbeiterzahl und Nutzung atypischer Beschäftigung ${ }^{7}$}

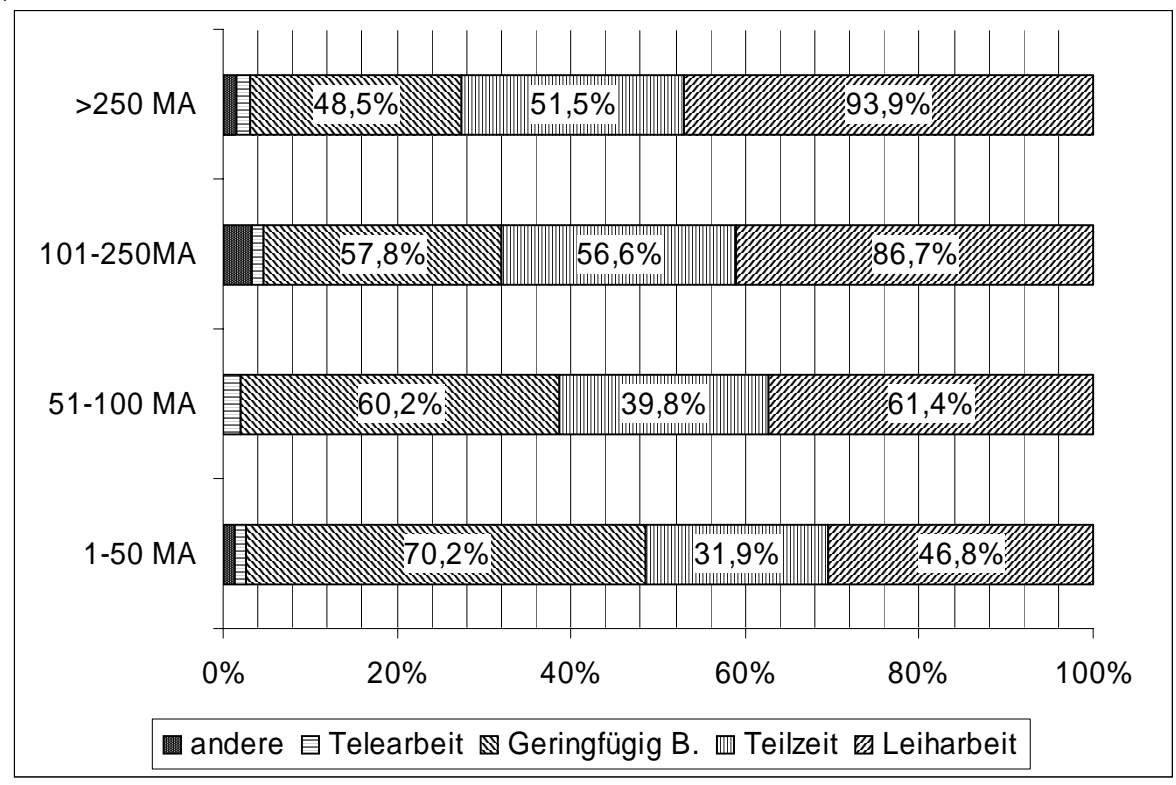

5 Somit weisen auch die Daten dieser Studie darauf hin, dass ein radikaler Wandel der Beschäftigungsformen in Deutschland nicht bestätigt werden kann (Bosch 2001, 224; Deutschmann 2002, 156).

$6 \quad$ 12,8\% der Betriebe machten keine Angaben zu diesem Punkt.

7 Die Unterschiede bei Leiharbeit, Teilzeitbeschäftigung sowie geringfügiger Beschäftigung sind signifikant, jedoch bei geringfügiger Beschäftigung nur auf dem 5\%-Niveau. 
Die vorliegende Untersuchung bestätigt, dass die Nutzung unterschiedlicher Instrumente zur Bewältigung von Produktionsschwankungen von der Betriebsgröße beeinflusst wird (Bellmann/Dahms/Wahse 2004, 34). Je mehr Mitarbeiter ein Unternehmen hat, desto höher ist der Anteil an Arbeitskräften einer Zeitarbeitsfirma, gleichzeitig nimmt die Zahl der geringfügig Beschäftigten ab. Hier bestätigt sich einerseits der Trend, dass Zeitarbeit mit steigender Betriebsgröße eine zunehmende Rolle spielt. Andererseits geht diese Zunahme zu Lasten der Nutzung geringfügig Beschäftigter.

\subsection{Nutzung in Abhängigkeit von der wirtschaftliche Lage}

Der Zusammenhang zwischen der Selbsteinschätzung der Unternehmen bezüglich ihrer wirtschaftlichen Lage und der Nutzungshäufigkeit verschiedener Formen atypischer Beschäftigung ist in Abbildung 2 dargestellt.

\section{Abb. 2: Wirtschaftliche Lage und Nutzung atypischer Beschäftigung ${ }^{8}$}

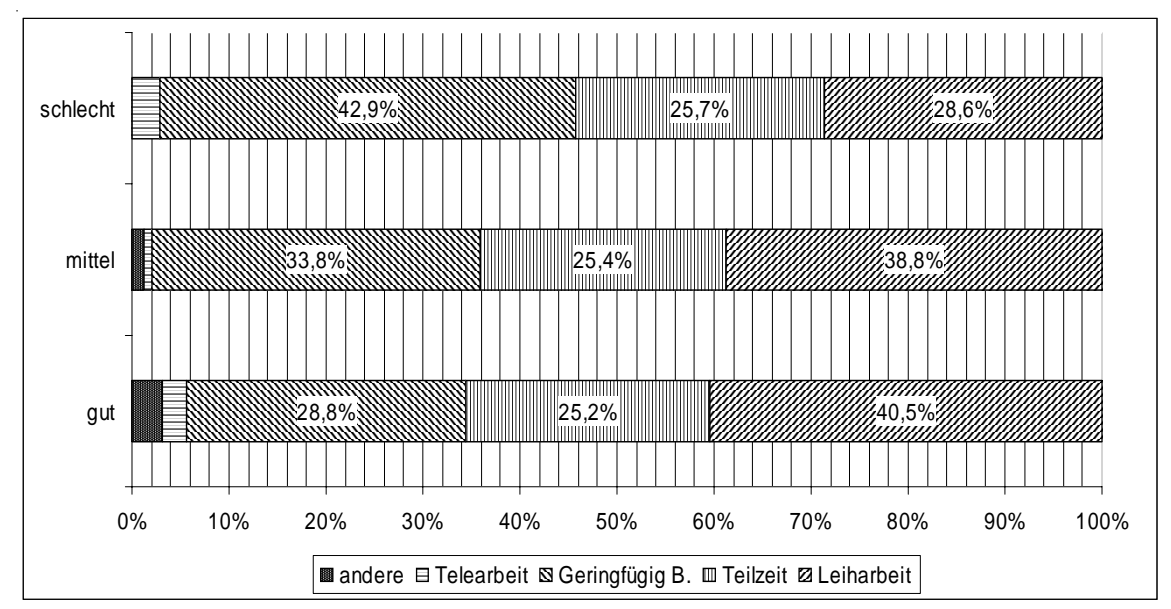

Je besser ein Unternehmen seine wirtschaftliche Lage einschätzt, desto mehr nimmt der prozentuale Anteil von geringfügigen Beschäftigungsverhältnissen ab und der Anteil der Leiharbeiter zu. Hier zeigt sich, dass die für den Arbeitgeber mit überdurchschnittlich hohen Kosten verbundene Leiharbeit (Klös 2000, 15) zunehmend stärker in Anspruch genommen wird, je besser es dem Unternehmen geht. In einer positiven wirtschaftlichen Lage werden gut ausgebildete Mitarbeiter benötigt, die dem Unternehmen über einen bestimmten Zeitraum ganztags zur Verfügung stehen. Dies lässt sich am ehesten mit Leiharbeit realisieren. Zudem werden die relativ hohen Kosten für Leiharbeit durch hohe Gewinne überkompensiert. Dagegen nutzen Unternehmen, die ihre wirtschaftliche Lage eher schlecht einschätzen, vermehrt geringfügig Beschäftigte. Diese sind im Vergleich zu Leiharbeitern relativ billig und können schnell wieder abgestoßen werden, wodurch Unternehmen in der angespannten Lage ihre Personalkosten auf niedrigem Niveau halten.

8 Signifikant sind nur die Unterschiede bei geringfügiger Beschäftigung. 


\subsection{Ziele der Nutzung}

Die Frage nach einer zielorientierten Einführung von Formen atypischer Beschäftigung bejahten 76,6\% der Unternehmen (nein 23,4 \%). Dies verdeutlicht, dass die Geschäftsleitungen der KMU sich mit atypischer Beschäftigung bestimmte Vorteile erhoffen. ${ }^{9}$

Die detaillierte Betrachtung der von den Unternehmen genannten Ziele zeigt, dass ökonomische Ziele, und dabei insbesondere die Steigerung der Produktivität (23,2\%) und Erhöhung der Flexibilität (59,7\%), überwiegen. Andere Ziele, wie z.B. die Erhöhung der Arbeitszufriedenheit oder die Verbesserung des Betriebsklimas wurden kaum angekreuzt. Die Dominanz ökonomischer Ziele ergibt sich auch, wenn man die verschiedenen Formen atypischer Beschäftigung gesondert betrachtet. Hierfür lassen sich drei mögliche Gründe anführen.

Erstens wird in den Ergebnissen das typische Antwortverhalten des betrieblichen Managements reflektiert, welches gegenüber Externen i.d.R. rational argumentiert und ökonomische bzw. Leistungsziele hervorhebt. Zudem kann dieses Antwortverhalten auch mit dem häufig ingenieurtechnischen Ausbildungs- und Berufshintergrund ostdeutscher Geschäftsleiter (Alt/ Lang 1998; Lang/Müller 2001) begründet werden.

Zweitens zeigt die geringe Nennung sozialer Ziele, wie Steigerung der Motivation oder Erhöhung der Zufriedenheit, dass solche Ziele nach Meinung der Geschäftsleitungen für atypische Beschäftigung kaum relevant sind. Primär werden diese Formen der Beschäftigung genutzt, um Kosten zu senken und Flexibilität zu erhöhen, wohingegen zur Erreichung sozialer Ziele andere Methoden (z.B. flexible Arbeitszeiten) sinnvoll erscheinen.

Drittens deutet die Hervorhebung ökonomischer Ziele darauf hin, dass atypische Beschäftigungsformen in den untersuchten Betrieben i.d.R. von betrieblicher Seite eingeführt werden. Der Mitarbeiterwunsch nach einer besseren Koordination von Arbeits- und Privatleben, ist aus dieser Perspektive eher selten der Grund für die Nutzung atypischer Beschäftigung im ostdeutschen Mittelstand.

94\% der befragten Unternehmensleitungen sind der Meinung, dass ihre mit atypischer Beschäftigung verbundenen Ziele im Großen und Ganzen erreicht wurden. 2,5\% waren nicht dieser Meinung, wobei die Gründe dafür nicht erhoben wurden. Einige Firmen (3,5\%) gaben an, dass die angestrebten Ziele nur zum Teil erreicht wurden. Hierbei wurden als Gründe die geringe Ausbildung, Qualifikation und damit Eignung der atypisch Beschäftigten, lange Anlernprozesse sowie die geringe Motivation angeführt, wobei diese Nennungen sich überwiegend auf Leiharbeit bezogen.

\subsection{Probleme der Nutzung}

Den angestrebten Zielen sowie deren Realisierung steht eine Reihe von Problemen gegenüber, die bei der Nutzung atypischer Beschäftigung in den KMU auftreten. Abbildung 3 verdeutlicht die genannten Probleme atypischer Beschäftigung über alle abgefragten Formen hinweg.

9 Die in diesem Zusammenhang zum Teil gestellte Frage, ob daraus ein strategisches Vorgehen der Unternehmen ableitbar ist (Mayne/Tregaskis/Brewster 2000, 72), kann auf Basis der vorliegenden Ergebnisse nicht beantwortet werden. 

Abb. 3: Probleme bei der Nutzung atypischer Beschäftigung
(Mehrfachnennungen möglich) ${ }^{10}$

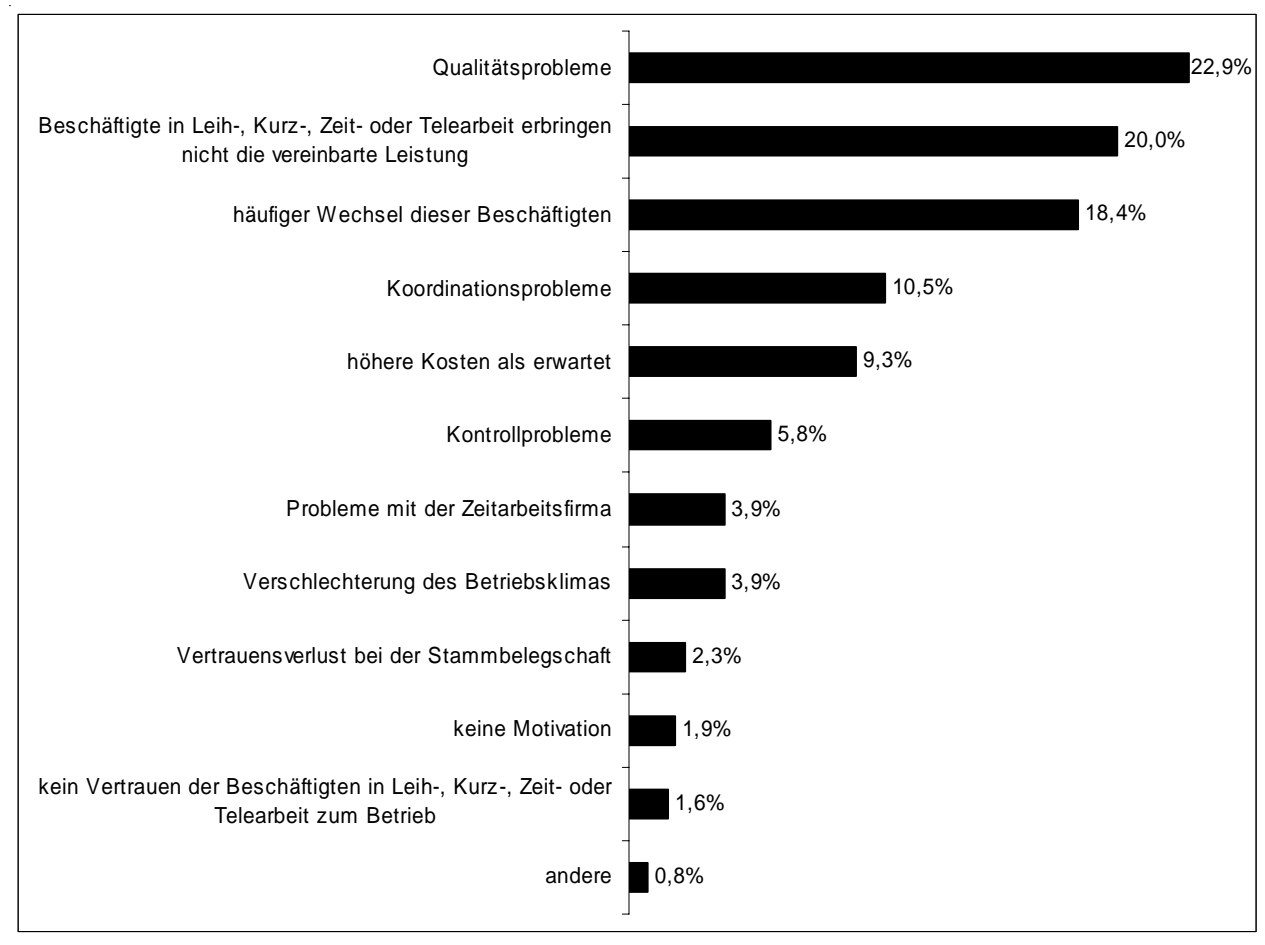

Wie schon bei der Betrachtung der Ziele zeigt sich eine deutliche Betonung von ökonomischen Problemen durch die Geschäftsleitungen, insbesondere in den Bereichen Koordination, Qualität und Leistung. In diese Richtung kann man auch die hohe Bedeutung des häufigen Wechsels atypisch Beschäftigter interpretieren. Da dieses Problem vom Management wahrgenommen wird, ist anzunehmen, dass auch hier ökonomische Aspekte, wie z.B. Anlernzeiten, dominieren. Die Konzentration der Wahrnehmung von ökonomischen Problemen verwundert indes nicht, wenn man berücksichtigt, dass die überwiegende Zahl der Unternehmen atypische Formen der Beschäftigung unter ökonomischen Prämissen eingeführt hat.

Die hohe Betonung von Leistungs- und Qualitätsproblemen lässt den Schluss zu, dass die Geschäftsleitungen offensichtlich erwarten, mit atypisch Beschäftigten „den richtigen Mitarbeiter für den richtigen Job“ zu erhalten. Atypisch Beschäftigte sollen von Anfang an entsprechende Leistung erbringen, eventuell notwendige betriebsspezifische Kenntnisse sowie Motivationsprobleme werden dabei nicht oder nur teilweise berücksichtigt.

Auf der Basis von 40 Fragebögen, die von Arbeitnehmervertretern sächsischer Betriebe der Metallbranche ausgefüllt wurden, werden nachfolgend die Aussagen der Geschäftsleitungen zu wahrgenommenen Problemen kontrastiert (vgl. Abbildung 4).

10 Die beiden Antworten in der Kategorie „andere“ waren „weite Anfahrtswege“ und „Probleme mit dem Betriebsrat“. 


\section{Abb. 4: Gegenüberstellung der Aussagen von Geschäftsleitungen und Arbeitnehmervertretern zu Problemen bei atypischer Beschäftigung (Mehrfachnennungen möglich) ${ }^{11}$}

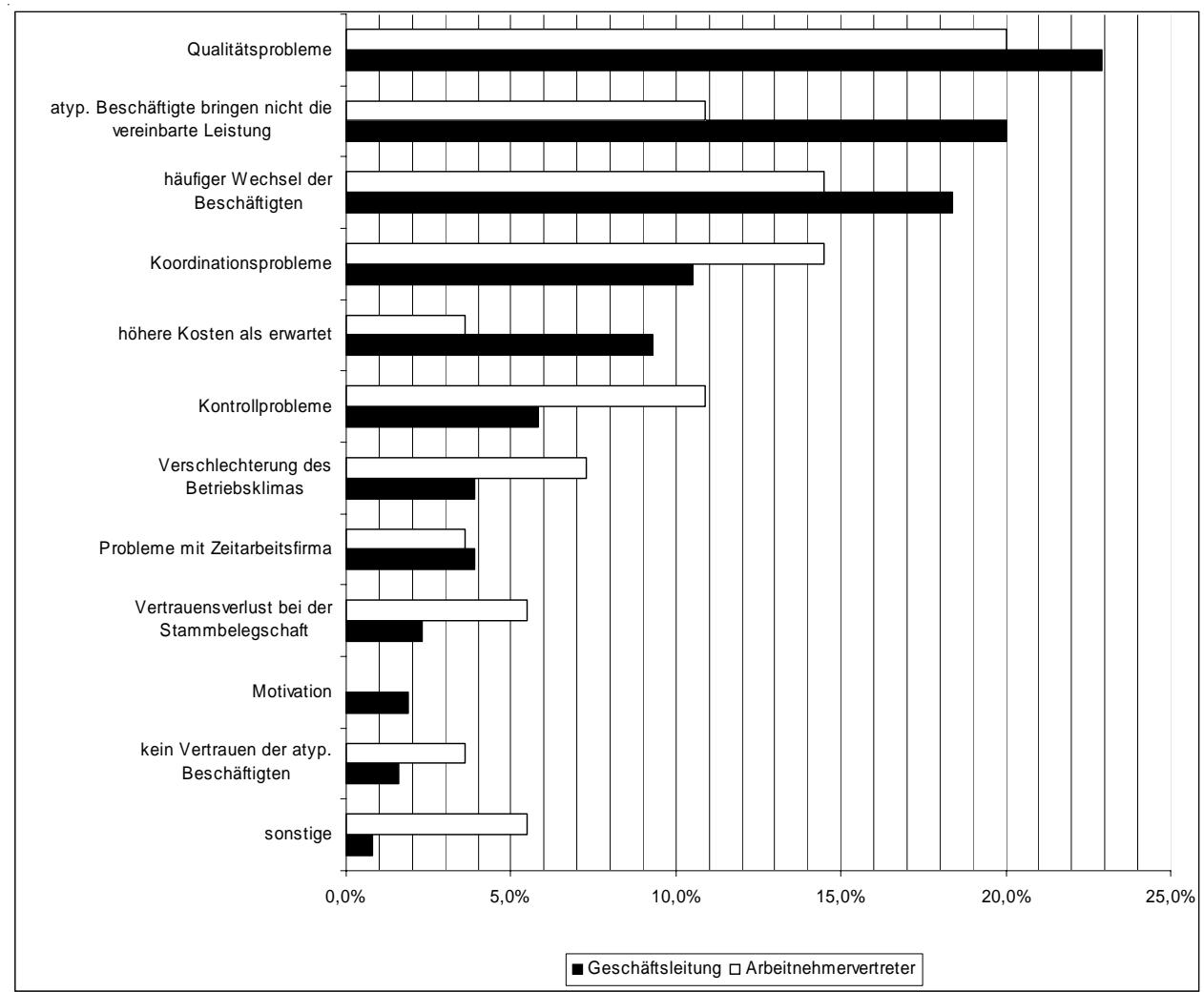

Aufgrund der geringen Fragebogenanzahl der Arbeitnehmervertreter sind die Antworten nur eingeschränkt vergleichbar. Dennoch lassen sich zwei Trendaussagen generieren.

Erstens betonen Arbeitnehmervertreter neben ökonomischen auch andere Probleme. Einerseits werden von ihnen verstärkt soziale Probleme, wie die Verschlechterung des Betriebsklimas oder Vertrauensverluste wahrgenommen. Durch ihren häufigen Kontakt zur Stammbelegschaft sowie zu atypisch Beschäftigten nehmen sie in größerem Umfang soziale Probleme auf der Mitarbeiterebene wahr. Andererseits betonen die Arbeitnehmervertreter auch stärker Koordinations- und Kontrollprobleme. Die häufig direkte Eingebundenheit der Arbeitnehmervertreter in den Herstellungsprozess der Betriebe lässt sie Probleme bei der Einarbeitung und Qualifikation atypisch Beschäftigter stärker wahrnehmen.

Zweitens bewerten die Arbeitnehmervertreter atypische Beschäftigung kritischer als die Geschäftsleitungen. Dies zeigt sich im Verhältnis der angeführten Probleme, das 2 (Geschäftsleitungen) zu 3 (Arbeitnehmervertreter) beträgt. Die Arbeitnehmervertreter haben im Gegen-

11 Als sonstige Probleme wurde von den Arbeitnehmervertretern die höhere Belastung der Stammbelegschaft durch ständiges Anlernen der Leiharbeiter genannt. Zudem existiert nach Meinung dieser Befragten das Problem, dass die gewünschte Leistung der atypisch Beschäftigten erst nach einer gewissen Einarbeitungszeit erreicht wird, da diese teilweise keine Vorkenntnisse über produktionsspezifische Zusammenhänge besitzen. 
satz zur Geschäftsleitung häufiger mit atypisch Beschäftigten in ihrer täglichen Arbeit zu tun, somit erleben sie öfter Probleme. Die kritischere Haltung der Arbeitnehmervertreter kann zudem damit zusammenhängen, dass sie mit der Nutzung atypischer Beschäftigung eine Art Erosion betrieblicher Mitbestimmung wahrnehmen, da atypisch Beschäftigte i.d.R. nicht unter Mitbestimmungsregelungen fallen.

\section{Zusammenfassung und Ausblick}

Atypische Beschäftigungsformen, insbesondere Leiharbeit, geringfügige Beschäftigung sowie Teilzeitarbeit, sind in ostdeutschen KMU etabliert. Atypische Beschäftigung wird dabei insbesondere von Seiten der Geschäftsleitung unter ökonomischen Gesichtspunkten eingeführt. Soziale Bedürfnisse der Mitarbeiter stehen hierbei im Hintergrund.

Mit der Nutzung atypischer Beschäftigungsformen im ostdeutschen Mittelstand ist eine Reihe von innerbetrieblichen Problemen verbunden. Das Management der KMU nimmt diese aus einer ökonomischen Perspektive wahr. Demgegenüber führen die befragten Arbeitnehmervertreter verstärkt soziale Probleme an, was insbesondere auf ihre allgemein kritische Haltung gegenüber solchen Beschäftigungsformen und auf ihre Erfahrungen mit atypischer Beschäftigung im operativen Betriebsprozess zurückzuführen ist.

Betrachtet man die Vielfältigkeit der Probleme, die vom Management und den Arbeitnehmervertretern der befragten KMU wahrgenommen werden, und berücksichtigt man, dass beim Umgang mit diesen eine Reihe von Aufwendungen und auch Kosten entstehen, so lässt sich zumindest die mit atypischer Beschäftigung angestrebte Kosteneinsparung anzweifeln. ${ }^{12}$

Dass vom Management der KMU trotz dieser Probleme eine positive Zielereichung konstatiert wird, hat insbesondere zwei Gründe:

Erstens werden die Kosten für die Problembewältigung nicht erfasst und nicht in betriebswirtschaftliche Rechnungen einbezogen. Somit erfolgt kein firmeninterner Vergleich zwischen Einsparungen bei Personalkosten und finanziellen Vorteilen erhöhter Flexibilität einerseits und Kosten für den Umgang mit auftretenden Problemen andererseits.

Zweitens erfährt das Management nur selektiv von Problemlagen im Zusammenhang mit atypisch Beschäftigten im Unternehmen, insbesondere wenn sie nur noch wenig direkten Kontakt zu den Mitarbeitern der unteren Ebene hat. Eine Reihe von Problemen, die mit atypischer Beschäftigung im Betrieb auftreten, wird direkt vor Ort gelöst und nicht immer der Geschäftsleitung berichtet.

Zusammenfassend kann man die Existenz eines, zumindest latenten, Spannungsverhältnisses vermuten. Atypische Beschäftigungsformen werden von Seiten der Geschäftsleitung eingeführt, um ökonomische Ziele zu erreichen. Dadurch entsteht jedoch eine Reihe von ökonomischen und sozialen Problemen, die vom Management nur selektiv wahrgenommen werden und die ein gutes Stück weit von den Mitarbeitern selbst bewältigt werden müssen.

Obwohl in der vorliegenden Untersuchung ostdeutsche KMU befragt wurden, lassen die Ergebnisse auch Rückschlüsse auf den gesamten Mittelstand zu. Insbesondere kann man davon ausgehen, dass KMU allgemein atypische Beschäftigung aufgrund zu erwartender ökonomischer Vorteile einführen, da einerseits solche Beschäftigungsformen im gesamten Unternehmens- und damit auch KMU-Sektor als wirksames Instrument gelten, aktuellen

12 Lautsch (2002) bemerkt allgemein, dass die Produktivitätseffekte atypischer Beschäftigung nicht klar sind. 
unternehmerischen Problemen zu begegnen, und andererseits haben die Geschäftsführer von KMU insgesamt einen technisch orientierten Ausbildungshintergrund(Mugler 1995, 20), was eher rationale Gründe für die Nutzung solcher Beschäftigungsformen erwarten lässt. Mitarbeiterwünsche nach flexibleren Regelungen, z.B. um Arbeit und Privatleben in einen besseren Einklang bringen zu können, sind aus dieser Perspektive eher die Ausnahme. Auch hinsichtlich auftretender Probleme können die Ergebnisse der Studie verallgemeinert werden. So ist insgesamt anzunehmen, dass die Geschäftsleitungen von KMU eher ökonomische Probleme als relevant interpretieren. Demgegenüber erfahren die Mitarbeiter der Stammbelegschaft, die mit atypisch Beschäftigten zusammenarbeiten, einerseits auch soziale Probleme, wie Unzufriedenheit oder fehlendes Vertrauen, andererseits nehmen sie auch verstärkt Koordinationsund Kontrollprobleme beim täglichen Umgang wahr.

Die Frage nach der KMU-Spezifik der in dieser Untersuchung genannten Probleme lässt sich auf Basis des vorliegenden Datenmaterials nicht beantworten. Hier besteht weiterer Forschungsbedarf. Einerseits muss nach den konkreten Ursachen für Probleme mit atypischer Beschäftigung gefragt werden. Hierzu ist jedoch ein umfassender Einblick in die Unternehmen, z.B. mit Hilfe von Betriebsfallstudien, erforderlich. Erst die Analyse der Problemursachen eröffnet die Möglichkeit, die in KMU auftretenden Probleme mit atypischer Beschäftigung vor dem Hintergrund der spezifischen innerbetrieblichen Abläufe und Sozialbeziehungen zu interpretieren. Eine solche Analyse könnte an Martin (2004) und die von ihm aufgezeigten unterschiedlich ausgeprägten Merkmale von Sozialbeziehungen in verschiedenen Beschäftigungsverhältnissen anschließen. Darüber hinaus erfordert die Beantwortung der Frage nach KMU-spezifischen Problemen mit atypischer Beschäftigung einen Vergleich mit großen Betrieben. Erst dadurch können größenklassenbezogene Unterschiede herausgearbeitet werden.

Ein weiteres zukünftiges Forschungsfeld ist der konkrete Umgang mit auftretenden Problemen. Insbesondere erscheint es relevant zu untersuchen, ob und wie die beteiligten Interessengruppen (Management, Stammbelegschaft, Arbeitnehmervertreter, atypisch Beschäftigte) versuchen, auftretende Probleme zu bewältigen.

\section{Literatur}

Alt, Ramona, Rainhart Lang (1997): Führungskräfte in sächsischen Klein- und Mittelbetrieben, Forschungsbericht zum Projekt: „Wandel von Wertorientierungen und Organisationsauffassungen von Führungskräften in sächsischen Klein- und Mittelbetrieben. Eine Untersuchung unter dem Aspekt des wirtschaftlichen Wiederaufbaus“. Chemnitz

Alt, Ramona, Rainhart Lang (1998): Wertorientierungen und Führungsverständnis von Managern in sächsischen Klein- und Mittelunternehmen; in: Rainhart Lang (Hg.): Führungskräfte im osteuropäischen Transformationsprozeß (Tagungsband des III. Chemnitzer Ostforums 1997). München/ Mering, 247-270

Becker, Cordelia (1998): Kollegen auf Zeit; in: managermagazin 36, 1, 104-110

Bellmann, Lutz u.a. (2004): IAB-Betriebspanel Ost. Ergebnisse der achten Welle 2003, Teil 2: Personalpolitik, Betriebliche Flexibilität, Weiterbildung; in: IAB-Forschungsbericht, Nr. 3/2004

Bosch, Gerhard (2001): Konturen eines neuen Normalarbeitsverhältnisses; in: WSI-Mitteilungen, 4, 219-230

Brewster, Chris u.a. (1994): Flexible working practises. The controversy and the evidence; in: Chris Brewster, Ariane Hegewisch (Hg.): Policy and practice in European human resource management. London/New York, 168-193 
Brinkmann, Ulrich (1996): Magere Bilanz: Neue Managementkonzepte (NMK) in transformierten ostdeutschen Betrieben; in: Markus Pohlmann, Rudi Schmidt (Hg.): Management in der ostdeutschen Industrie. Opladen, 215-248

Brinkmann, Ulrich (2003): Die Labormaus des Westens: Ostdeutschland als Vorwegnahme des Neuen Produktionsmodells?; in: Klaus Dörre, Bernd Röttger (Hg.): Das neue Marktregime. Konturen eines nachfordistischen Produktionsmodells. Hamburg, 250-269

Cardon, Melissa (2003): Contingent Labour as an Enabler of Entrepreneurial Growth; in: Human Resource Management 42, 4, 357-373

Corral, Antonio, Inigo Isusi (2003): Part-time work in Europe, European Foundation for the Improvement of Living and Working Conditions. Dublin

Deutschmann, Christoph (2002): Postindustrielle Industriesoziologie. Weinheim/München

Dimitrova, Dimitrina (2003): Controlling teleworkers: supervision and flexibility revisited; in: New Technology, Work and Employment 18, 3, 181-195

Düll, Herbert, Peter Ellguth (1999): Betriebliche Strukturen der Teilzeitbeschäftigung in West- und Ostdeutschland; in: MittAB 32, 3, 269-280

Foote, David A., Timothy B. Folta, (2002): Temporary workers as real options; in: Human Resource Management Review 11, 181-208

Grimm, Natalie (2004): Flexibilität durch Leiharbeit: Zumutung oder Chance?; in: SOFI-Mitteilungen, 32, 129-139

Hoffmann, Edeltraut, Ulrich Walwei (1998): Normalarbeitsverhältnis: ein Auslaufmodell?; in: MittAB 31, 3, 409-425

IAB-Betriebspanel Ost (2003): Ergebnisse der siebten Welle 2002, SÖSTRA Sozialökonomische Strukturanalyse GmbH Berlin

Klös, Hans-Peter (2000): Zeitarbeit - Entwicklungstrends und arbeitsmarktpolitische Bedeutung; in: iw-trends 27, 1, 5-21

Krömmelbein, Silvia (2000): Bedeutung der Telearbeit für Beschäftigung und Arbeitsmarkt am Beispiel der Region Rhein-Main aus betrieblicher Sicht; in: MittAB 33, 1, 136-153

Lang, Rainhart, Sven Müller (2001): Privatisation, Perception of Success and Attitudes of Managers in the East German Transformation Process; in: Kari Liuhto (Hg.): Ten Years of Economic Transformation. Volume III: Societies and Institutions in Transition. Studies in Industrial Engineering and Management, Number 16. Lappeenranta, 391-407.

Lautsch, Brenda A. (2002): Uncovering and explaining variance in the features and outcomes of contingent work; in: Industrial and Labour Relations Review 56, 23-43

Martin, Albert (2002): Selbständige Arbeitnehmer oder abhängige Selbständige; in: Albert Martin, Werner Nienhüser (Hg.): Neue Formen der Beschäftigung - neue Personalpolitik? München/ Mering, 17-60

Martin, Albert (2004): Formen der Beschäftigung. Empirische Besonderheiten der sozialen Beziehung zum Arbeitgeber; in: Schriften aus dem Institut für Mittelstandsforschung, Heft 23, Lüneburg

Martin, Albert/Nienhüser, Werner (2002): Neue Formen der Beschäftigung; in: Albert Martin, Werner Nienhüser (Hg.): Neue Formen der Beschäftigung - neue Personalpolitik? München/Mering, 1-16

Matusik, Sharon F., Charles W. L. Hill (1998): The utilization of contingent work, knowledge creation, and competitive advantage; in: Academy of Management Review 23, 4, 608-697

Mayne, Lesley u.a. (2000): A Comparative Analysis of the Link Between Flexibility and HRM Strategy; in: Chris Brewster, Wolfgang Mayrhofer, Michael Morley (Hg.): New Challenges for European Human Resource Management. London, 72-96

Meinerz, Klaus Peter (1996): Einstellungen, Werthaltungen und Leitbilder von Managern in Ostdeutschland. Kognitive und normative Voraussetzungen der Rekonstruktion betrieblicher Sozialbeziehungen; in: Markus Pohlmann, Rudi Schmidt (Hg.): Management in der ostdeutschen Industrie. Opladen, 177-216

Mugler, Josef (1995): Betriebswirtschaftslehre der Klein- und Mittelbetriebe. Wien 
Nienhüser, Werner, Walter Baumhus (2002): Fremd im Betrieb; in: Albert Martin, Werner Nienhüser (Hg.): Neue Formen der Beschäftigung - neue Personalpolitik? München/Mering, 61-120

Bertelsmann Stiftung, Hans-Böckler-Stiftung (Hg.) (1998): Mitbestimmung und neue Unternehmenskulturen. Bilanz und Perspektiven. Gütersloh

Pearce, Jone L. (1993): Toward an organizational behavior of contract laborers: Their psychological involvement and effects on employee co-workers; in: Academy of Management Journal 36, 5, 1082 1096

Rudolph, Helmut, Esther Schröder (1997): Arbeitnehmerüberlassung: Trends und Einsatzlogik; in: MittAB 30, 1, 102-126

Schäfer, Holger (2000): Atypische Beschäftigung. Entwicklungstrends und Bedeutung für den Arbeitsmarkt; in: iw-trends 27, 4, 41-54

Seifert, Matthias, Peter Pawlowsky (1998): Innerbetriebliches Vertrauen als Verbreitungsgrenze atypischer Beschäftigungsformen; in: MittAB 31, 3, 599-611

Tilly, Chris (1992): Dualism in part-time employment; in: Industrial Relations 31, 2, 330-347

Topi, Heikki (2004): Supporting Telework: Obstacles and solutions; in: Information Systems Management 21, 3, 79-86

Vogel, Berthold (1999): Arbeitslosigkeit in Ostdeutschland. Konsequenzen für das Sozialgefüge und für die Wahrnehmung des gesellschaftlichen Wandels; in: SOFI-Mitteilungen, 27, 15-23

Voss-Dahm, Dorothea (2004): Geringfügige Beschäftigung und Segmentation auf innerbetrieblichen Arbeitsmärkten des Einzelhandels; in: ARBEIT, 13, 4, 354-367

Anschrift des Verfassers:

Dr. Ingo Winkler

TU Chemnitz

Fakultät für Wirtschaftswissenschaften

Professur für Organisation und Arbeitswissenschaft

D-09107 Chemnitz

ingo.winkler@wirtschaft.tu-chemnitz.de

\section{Schlagwörter: Personal, regional, Zukunft der Arbeit}

